\title{
LA COMPETENCIA CIENTÍFICA EN LOS ESTUDIOS DE BACHILLERATO EN ESPAÑA: UN ANÁLISIS CURRICULAR RETROSPECTIVO
}

\author{
Luis Erneta Altarriba* y María Casas Jericóo*
}

\section{RESUMEN}

En las últimas décadas la difusión e implicación que la ciencia tiene en la sociedad ha favorecido que la educación en materia científica se haya convertido en un objetivo general del aprendizaje en el marco europeo y espańol. Se persigue que los alumnos aprendan contenidos científicos, pero también que desarrollen destrezas y habilidades científicas. En España, la competencia científica se incluyó oficial y explícitamente en el currículum con la Ley Orgánica de Educación (LOE, 2006) y volvió a ponerse en valor con la Ley Orgánica para la Mejora de la Calidad Educativa (LOMCE, 2013). El objetivo de esta investigación es el análisis retrospectivo, desde una perspectiva curricular, de la transición LOE-LOMCE en la oferta de nuevas materias que favorecen el desarrollo de la competencia científica en Bachillerato. Los resultados muestran que, si bien en la LOMCE aumentó la oferta de materias para el desarrollo de competencias científicas, la posibilidad real de que el alumnado curse dichas materias es menor, dado que su carácter optativo no siempre asegura su impartición. PALABRAS ClAVE: análisis curricular, competencia científica, cultura científica, alfabetización científica, Bachillerato, estudios preuniversitarios.

\section{THE SCIENTIFIC COMPETENCE ON THE SPANISH BACCALAUREATE: A RETROSPECTIVE STUDY OF CURRICULA}

\section{Abstract}

During the past decades, the increasing presence of Science in the society has fostered the scientific issue to become a general aim for education in the European context. Students should not only learn scientific contents but also develop the scientific skills at school. In Spain, the scientific competence was officially included in the curriculum of "LOE" educational law (approved in 2006) and it remains in the "LOMCE" context (2013). In this research, a comparative analysis of "LOE" and "LOMCE" curricula was conducted to study how the educational law transition (from LOE to LOMCE) affected the offer of subjects that foster the scientific competence of Baccalaureate students. Results show that the offer of subjects that may favor the scientific competence is higher in the "LOMCE"context. However, the students' likelihood of taking these subjects is low because they are not mandatory.

KEYWORDs: curricular analysis, scientific competence, scientific culture, scientific literacy, Baccalaureate. 


\section{INTRODUCCIÓN}

En las últimas décadas, la sociedad occidental ha presenciado un desarrollo científico y tecnológico sin precedentes, cuyas consecuencias no siempre han sido positivas a escala global. El cambio climático, la contaminación de aguas, suelos y atmósfera, la deforestación, los desequilibrios sociales, las guerras o el hambre son algunos de los problemas ambientales y sociales a los que se enfrenta hoy la humanidad (IPPC, 2015; PNUD, 2016; PNUMA, 2012). Para hacerles frente, la sociedad tiene por delante una ardua y amplia tarea que varía desde entender cómo se regula el medio ambiente hasta cuáles son los avances del desarrollo científico y tecnológico en ámbitos tan específicos como la genómica, los nuevos materiales o las energías renovables.

Resulta preciso, por tanto, que la ciudadanía reciba una alfabetización científica, al mismo tiempo que desarrolle habilidades prácticas y adquiera una serie de valores que permitan un juicio crítico que favorezcan la convivencia entre las personas y entre estas y su medio. Este triple aprendizaje de contenidos, habilidades y valores, cuyo objetivo es la formación de ciudadanos responsables, tiene lugar, en gran medida, en la escuela y ha ido adquiriendo especial importancia y peso en los currículos durante las dos últimas décadas.

Paralelamente, en este tiempo la educación ha ido cambiando, desarrollando y aplicando nuevas metodologías y recursos pedagógicos para dar respuesta a las demandas sociales del momento. A nivel europeo, uno de los cambios educativos más importantes de los últimos años fue la inclusión curricular del aprendizaje competencial. En respuesta a las recomendaciones de la Unión Europea, en el contexto español, la Ley Orgánica de Educación (en adelante, LOE), aprobada en 2006, definió ocho competencias que el alumnado debía haber desarrollado al término de la escolaridad obligatoria, siendo recomendable su continuidad durante el Bachillerato (etapa puente entre el periodo obligatorio y el universitario o profesionalizante). Entre estas competencias se encuentra la llamada competencia científica, objeto de estudio de esta investigación.

El objetivo del presente estudio es analizar qué materias del Bachillerato han sido y son las que contribuyen a que los alumnos desarrollen la competencia científica experimental. En primer lugar, con el objeto de contextualizar la investigación y conocer los antecedentes, se recogen algunas de las iniciativas pioneras del marco curricular de la Ley General de Ordenación del Sistema Educativo (LOGSE), para, posteriormente, estudiar las aportaciones de la LOE y la actual Ley Orgánica para la Mejora de la Calidad Educativa (LOMCE). En segundo lugar, se desglosan con mayor detalle las materias del contexto normativo actual, realizando tanto una revisión de sus contenidos como un análisis crítico de la probabilidad de que estas sean finalmente ofertadas en los centros educativos.

* Departamento de Educación, Gobierno de Navarra.E-mail: lernetaa@eduacion.navarra.es.

** Departamento de Biología Ambiental, Facultad de Ciencias, Universidad de Navarra, España. E-mail: mcasas.1@alumni.unav.es. 


\section{FUNDAMENTACIÓN TEÓRICA: ANTECEDENTES Y SITUACIÓN ACTUAL DE LA COMPETENCIA CIENTÍFICA EN LOS CURRÍCULOS DEL SISTEMA EDUCATIVO ESPAÑOL}

Los actuales retos ambientales y sociales demandan una sociedad activa, responsable, crítica, formada y, en definitiva, capacitada para hacerles frente de forma efectiva. En este sentido, la educación se ha perfilado tradicionalmente como una herramienta esencial para promover cambios de valores y actitudes en la ciudadanía, los cuales, en última instancia, se traducirían en un cambio generalizado de comportamiento que quedaría impreso en la cultura (Puig y Casas, 2017). Para lograr este ambicioso objetivo, en las últimas décadas se han formulado políticas y estrategias internacionales en materia de educación para alcanzar un modelo de desarrollo sostenible y justo tanto desde el punto de vista social como ambiental.

Una de las iniciativas más importantes en materia de educación a nivel internacional fue el Decenio de las Naciones Unidas de la Educación con miras para el Desarrollo Sostenible (2005-2014), un plan estratégico cuyo objetivo general era «(...) integrar los principios, valores y prácticas del desarrollo sostenible en todas las facetas de la educación y el aprendizaje» (UNESCO, 2006, p. 6). En el contexto del Decenio, la Comisión Económica de la ONU para Europa (CEPE) desarrolló una estrategia de acción específica para esta región. Siete años después, en 2012, este organismo publicó "Aprender para el futuro", un documento donde se establecen cuatro competencias básicas para la educación para el desarrollo sostenible -aprender a conocer, aprender a hacer, aprender a ser y aprender a vivir juntos (CEPE, 2012)-. Y un año más tarde vio la luz "Capacitar a los Educadores para un Futuro Sostenible» (CEPE, 2013), un documento específico para la capacitación profesional de los educadores.

Paralelamente, en el periodo descrito, desde la Unión Europea se han dictado también políticas educativas en las que se aboga por la formación conceptual y el desarrollo de destrezas científicas. Así quedaba reflejado en las palabras de su comisario de educación: «El conocimiento científico está llamado a desempeñar un papel cada vez más importante en el debate público, en la toma de decisiones y en la legislación» (Pedrinaci, 2011, p. 196). En esta misma línea, el informe Rocard, desarrollado por un grupo de expertos en educación científica, también recogió la idea de la urgencia y necesidad de pasar a la acción con rapidez para favorecer el desarrollo educativo de este tipo de competencias por su implicación en el futuro económico y social en el contexto europeo (Rocard et al., 2008).

La necesidad de una formación científica experimental, impresa en el marco internacional y el europeo, se ha ido materializando en las dos últimas décadas en el ámbito educativo formal español mediante la creciente introducción de contenidos y materias de naturaleza ambiental, tecnológica y biosanitaria. En este contexto, a continuación, se analiza cómo se ha plasmado la competencia científica en cuatro materias curriculares del Bachillerato pertenecientes a las tres últimas grandes reformas del sistema educativo español. Se trata de materias novedosas, que aparecieron por primera vez en su correspondiente contexto normativo y son Ciencia, Tecnología y Sociedad (LOGSE), Ciencias para el Mundo Contemporáneo (LOE), Cultura Científica y Anatomía Aplicada (LOMCE) (tabla 1). 


\begin{tabular}{|c|c|c|c|c|}
\hline \multicolumn{5}{|c|}{$\begin{array}{l}\text { TABLA 1. NUEVAS MATERIAS DE } 1 .^{\circ} \text { DE BACHILLERATO AFINES A LA } \\
\text { COMPETENCIA CIENTÍFICA EN LA LOGSE, LA LOE Y LA LOMCE }\end{array}$} \\
\hline MarCo LEGAL & $\begin{array}{c}\text { LOGSE } \\
\text { (RESOLUCIÓN DEL } \\
\text { 29/11/1992) }\end{array}$ & $\begin{array}{l}\text { LOE } \\
(\mathrm{RD} 1467 / 2007)\end{array}$ & $\begin{array}{r}\mathrm{LO} \\
(\mathrm{RD} 11\end{array}$ & $\begin{array}{l}1 \mathrm{CE} \\
5 / 2014)\end{array}$ \\
\hline Nombre de las materias: & $\begin{array}{l}\text { Ciencia, Tecnología } \\
\text { y Sociedad (CTS) }\end{array}$ & $\begin{array}{l}\text { Ciencias para el Mundo } \\
\text { Contemporáneo } \\
(\mathrm{CMC})\end{array}$ & $\begin{array}{l}\text { Cultura Cien- } \\
\text { tífica (CC) }\end{array}$ & $\begin{array}{l}\text { Anatomía } \\
\text { Aplicada } \\
\text { (AA) }\end{array}$ \\
\hline Carácter de las materias: & Materia optativa & Materia obligatoria & $\begin{array}{l}\text { Materia } \\
\text { optativa* }^{*}\end{array}$ & $\begin{array}{l}\text { Materia } \\
\text { optativa* }^{*}\end{array}$ \\
\hline $\begin{array}{l}\text { Oferta de las materias } \\
\text { según itinerarios: }\end{array}$ & $\begin{array}{l}\text { En los itinerarios } \\
\text { «Tecnológico» y de } \\
\text { «Humanidades y } \\
\text { Ciencias Sociales» }\end{array}$ & En todos los itinerarios & $\begin{array}{l}\text { En todos los } \\
\text { itinerarios }\end{array}$ & $\begin{array}{l}\text { En todos los } \\
\text { itinerarios }\end{array}$ \\
\hline
\end{tabular}

* Materia a escoger del bloque de asignaturas específicas. Fuente: elaboración propia.

Indudablemente, en los currículos de Bachillerato de estas leyes educativas ha habido, y hay, otras materias que abordan el desarrollo de la competencia científica, como son Biología y Geología, Física y Química, Matemáticas, Ciencias de la Tierra y del Medio Ambiente o incluso Dibujo Técnico o Tecnología Industrial. Sin embargo, este estudio se centra únicamente en las nuevas materias de cada contexto normativo, que desde un enfoque divulgativo contribuyen a avanzar, ampliar y diversificar el desarrollo del aprendizaje competencial de forma global independientemente del itinerario escogido.

\section{La Ley General de Ordenación del Sistema Educativo}

La LOGSE, aprobada en 1990, introdujo importantes cambios en el sistema escolar en respuesta a las demandas sociales y culturales del momento. El establecimiento de tres niveles de enseñanza (Educación Infantil, Primaria y Secundaria) y la reorganización del currículo fueron dos de los cambios más relevantes que implantó esta ley $(B O E, 1990)$.

En el currículo de la LOGSE los contenidos científicos ganaron peso, hecho que Gómez (2001) atribuyó a la preocupación y sensibilidad social que existía en el momento por los problemas ecológicos. En esta línea, se actualizaron los contenidos de las materias y se crearon nuevas asignaturas. Entre estas nuevas materias cabe destacar una optativa de $10^{\circ}$ de Bachillerato llamada Ciencia, Tecnología y Sociedad (en adelante, CTS).

La CTS fue una materia arriesgada y novedosa (Acevedo, 1996; Solbes y Vilches, 2002; Díaz y García, 2011), que seguía la estela de experiencias y demandas similares acontecidas en los contextos anglosajón y germano (Vilches, 1994; Acevedo, 1997). Diseñada con un enfoque constructivista e innovador, la CTS pretendía aunar los planteamientos propios de las ciencias experimentales y la potencialidad de la ciencia tecnológica con sus implicaciones sociales y éticas, motivo por el 
cual fue la materia de Filosofía, con su método, la considerada como herramienta esencial y el eje vertebrador de la misma (Membiela, 2001). Por este motivo, gran parte de los contenidos de CTS tenían un carácter humanístico.

En realidad, el contenido de la CTS no fue tratado solo individualmente como una materia específica, sino también como parte de una metodología propositiva de integración de los aspectos de índole científica y/o tecnológica en la sociedad, de modo que tuvo cierto grado de penetración en otras materias planteadas por el currículo de la LOGSE.

Para cubrir los objetivos propios de la materia de CTS se empleaba el desarrollo de unidades didácticas en las que los contenidos eran expuestos desde un enfoque práctico que procurara responder a cuestiones de interés social (Álvarez y Fernánez-Posse, 1996; Arroyo, 1999). Pese a su evidente singularidad y atractivo, casi una década después de la implantación de la LOGSE, la CTS era calificada como «desconocida por gran parte del profesorado» (Acevedo, 2004, pp. 10-11). Es posible que su escaso recorrido se debiera en parte a que se trataba de una optativa de $1 .^{\circ}$ de Bachillerato, un curso complejo, cargado de contenidos y con una amplia oferta de materias optativas instrumentales, algunas de ellas incluso excluyentes o determinantes para la elección de estudios superiores (López, 1998). Una reflexión muy vinculada, como se verá más adelante, a las condiciones de los diferentes marcos curriculares.

\section{La Ley Orgánica de Educación}

La LOE, aprobada en el año 2006, introdujo, siguiendo las recomendaciones de la Unión Europea (2006/962/CE), las llamadas competencias básicas para las etapas de Educación Primaria y Secundaria Obligatorias (BOE, 2006a). Un concepto con un relativo éxito docente que ha marcado las metodologías en los últimos años. Sin embargo, para el periodo educativo aquí analizado -el Bachilleratolas competencias se perfilaron recomendables, aunque no obligatorias, al contrario de lo que sucedía en las etapas previas. Las competencias básicas se definieron en el anexo I de los currículos de la enseñanza obligatoria como aquellas

[...] que debe haber desarrollado un joven o una joven al finalizar la enseńanza obligatoria para poder lograr su realización personal, ejercer la ciudadanía activa, incorporarse a la vida adulta de manera satisfactoria y ser capaz de desarrollar un aprendizaje permanente a lo largo de la vida (BOE, 2006b, 2007a).

Aunque fueron ocho las competencias recogidas en la LOE, en el caso que nos ocupa se ha fijado la atención en la comúnmente llamada competencia científica, que formalmente se denominó «Competencia en el conocimiento y la interacción con el mundo físico». Esta hacía referencia al desarrollo de habilidades que permitieran al alumno

[...] desenvolverse adecuadamente, con autonomía e iniciativa personal en ámbitos de la vida y del conocimiento muy diversos (salud, actividad productiva, consumo, 
ciencia, procesos tecnológicos, etc.) y para interpretar el mundo, lo que exige la aplicación de los conceptos y principios básicos que permiten el análisis de los fenómenos desde los diferentes campos de conocimiento científico involucrados (anexo I, BOE, 2006b, 2007a).

Al desarrollo de la competencia científica se contribuía desde las diferentes materias recogidas en el currículo, aunque es evidente, dado el enfoque tomado, que las de carácter científico-experimental eran más idóneas para alcanzar dicho objetivo. En este sentido, cabe destacar la aparición de Ciencias para el Mundo Contemporáneo (en adelante, $\mathrm{CMC}$ ), una materia obligatoria para $10^{\circ}$ de Bachillerato que constituyó una clara oportunidad para potenciar el tratamiento de la competencia científica (Predrinaci, 2006, 2008; Redondo y Gómez, 2006; Tárraga y De Pro, 2013) en una etapa educativa, el Bachillerato, para la que, a priori, el desarrollo de competencias no era obligatorio.

Las principales características que llevan a destacar la adecuación de CMC para el aprendizaje competencial son cuatro:

1) La heterogeneidad del alumnado que la cursaba. Al tratarse de una materia obligatoria, en CMC confluían alumnos de todas las modalidades de Bachillerato. Este hecho era muy enriquecedor para el alumnado, ya que permitía el intercambio de opiniones sobre un mismo tema desde enfoques diferentes. De este modo, además, se contribuía a dejar atrás la tradicional dicotomía entre las «Ciencias» y las «Letras» (Martín, Nieda y Pérez, 2008).

2) La variedad y aplicabilidad de los contenidos recogidos por la materia, que incluían aspectos de gran impacto social muy presentes en la realidad cotidiana del alumnado. Se trataba de cuestiones muy ligadas al enfoque de la investigación y resolución de problemas, como son el estudio de las enfermedades infecciosas, los beneficios y riesgos de la biotecnología, el impacto ambiental de las actividades humanas o la tipología de los nuevos materiales tecnológicos empleados en la vida cotidiana del ciudadano (BOE, 2007b, anexo I).

3) La oportunidad de introducir conceptos novedosos aportados desde diferentes disciplinas con un enfoque global. Este era el caso, por ejemplo, del estudio de la utilización de los transgénicos, un avance tecnológico actual de gran complejidad conceptual que requiere tanto la explicación tanto de aspectos de la biología molecular como el planteamiento de determinadas cuestiones éticas y morales de amplio calado social, económico y medioambiental. A pesar de ello, en CMC no se apreciaba una clara confluencia de los enfoques humanístico y científico, un aspecto de gran importancia que, como se ha comentado, sí se vio reflejado en la materia CTS de la LOGSE.

4) El empleo de herramientas metodológicas prácticas con una clara vocación competencial. Este hecho fue plasmado claramente por la mayoría de los manuales de texto distribuidos por las editoriales en Espańa (De Pro, 2009). En ellos se incluían de forma habitual actividades dirigidas al desarrollo de las competencias, como, por ejemplo, la realización de un trabajo de investigación en grupo, el análisis de noticias de prensa, el visionado de vídeos 
documentales específicos o el planteamiento de debates sobre temas de actualidad (cambio climático, impacto ambiental, empleo de transgénicos, terapia génica, etc.).

La novedosa CMC fue muy aplaudida y apoyada por la comunidad educativa afín a las ciencias experimentales (Fernández-González, 2008). En palabras de Tárraga y De Pro (2013, p. 24):

El nacimiento de esta asignatura fue esperanzador: llevaba a las aulas temáticas de gran actualidad, sugería una forma diferente de enseñar ciencias, ampliaba las posibilidades de alfabetización científica a estudiantes que no iban a seguir los estudios en ciencias o tecnologías.

Suponía, en definitiva, un paso adelante ante las carencias y necesidades observadas en este tipo de conocimientos en el alumnado de la etapa.

Sin embargo, y pese a los argumentos expuestos, no todas las cuestiones planteadas en CMC fueron positivas ni sencillas. La puesta en marcha de esta materia, tal y como recoge Fernández-González (2008), tuvo múltiples objeciones, en su mayoría planteadas por un profesorado poco preparado para el enfoque y los contenidos de esta. Las inquietudes y reticencias que manifestaban los docentes, ampliamente descritas por el autor (2008, pp. 191-194), podrían sintetizarse en cuatro:

1) los contenidos se disponían en un programa de poco nivel al ser abordado desde una perspectiva interdisciplinar;

2) la formación específica del profesorado en temas de excesiva actualidad era escasa;

3) la descripción de las leyes fundamentales podía ser sustituida por debates y discusiones sin un sustrato de conocimiento real;

4) la ausencia de herramientas didácticas metodológicas realmente adaptadas a este tipo de contenidos y objetivos de carácter divulgador.

Los nuevos planteamientos que vieron nacer la CMC supusieron, en cierto modo, un enfoque más positivo ante el escenario poco afable dibujado por las estadísticas e informes de especialistas alertados por la evidente reducción en el número de alumnos matriculados en estudios de índole científica tanto en España como en Europa. Fernández-González (2008) y Pedrinaci (2012), al describir el contexto del nacimiento de la materia de CMC, recapitulan los posibles motivos causantes de dicha reducción de estudiantes y los describían de forma genérica como indicadores de una crisis en las ciencias. Algunos de los motivos recogidos por estos autores se referían al hecho de que los programas formativos estuvieran sobrecargados, que la mayoría de los contenidos tuvieran su origen en el siglo pasado, y que implicaran procedimientos con un alto grado de abstracción o que carecieran de conexión con situaciones de la vida real. Todas estas cuestiones incidían disminuyendo el interés del alumnado por las materias y dificultando el posicionamiento de dichos estudios como vías reales para la adquisición de conocimientos útiles en el mundo laboral (Gil y Vilches, 2001). 
La experiencia espańola no fue aislada; paralelamente al nacimiento de la CMC, otros países de Europa también incorporaron una materia de este tipo en sus currículos oficiales. Es el caso de «Science for Public Understanding» en Gran Bretaña-obligatoria para todos los alumnos en el «A/AS Level» (educación preuniversitaria) - o de «Enseignement scientifique, série littérarie» en Francia -obligatoria únicamente para los itinerarios de letras de «Première» (educación secundaria)(Mas, 2006; Millar y Hunt, 2006; Predrinaci, 2006).

\section{Ley para la Mejora de la Calidad Educativa}

La LOMCE, aprobada en 2013, definió siete competencias -rebautizadas, siguiendo la nomenclatura del espacio Europeo (Comisión Europea, 2012), como "Competencias clave»- que, al igual que sucedía en el caso de la LOE, los alumnos deberían haber adquirido al término de la escolarización obligatoria (BOE, 2015a). En este caso, la competencia científica se describe bajo el título de "Competencia matemática y competencias básicas en ciencia y tecnología»-afinando quizás un poco más en el ámbito experimental de la dicha competencia-y se definió de la siguiente manera:

Son aquellas que proporcionan un acercamiento al mundo físico y a la interacción responsable con él desde acciones, tanto individuales como colectivas, orientadas a la conservación y mejora del medio natural, decisivas para la protección y mantenimiento de la calidad de vida y el progreso de los pueblos (BOE, 2015b, p. 6994).

Nuevamente la LOMCE muestra una especial sensibilidad sobre la cuestión científica, hecho que queda reflejado no solo en la definición de las competencias clave, sino también en el empleo. Ya en el preámbulo de la ley los resultados de la última evaluación externa PISA (Programa Internacional de Evaluación de los Alumnos) (OCDE, 2011) se emplearon como argumento para potenciar la difusión de las cuestiones científicas. Sin embargo, en contraposición, el desarrollo de las competencias vuelve a ser recomendable, pero no obligatorio, para la etapa de Bachillerato.

En este contexto, las materias de la LOMCE que podrían considerarse herederas de CMC (LOE) son dos: Cultura Científica (en adelante, CC) y Anatomía Aplicada (AA), ambas optativas de $1 .^{\circ}$ de Bachillerato. Si bien la aparición de dos materias en las que se fomenta específicamente la competencia científica es un hecho a destacar muy positivamente, la vuelta a un régimen de optatividad implica que el alumnado de algunos itinerarios pueda no llegar a adquirir ningún conocimiento próximo a las ciencias experimentales aplicadas. Esta cuestión se discutirá en profundidad más adelante.

Por último, es preciso apuntar que la importancia que la LOMCE otorga al desarrollo de la competencia científica también se ve reflejado en $4 .^{\circ}$ de ESO, con la aparición de otras dos materias: Cultura Científica-que, pese a compartir el nombre con la materia de $1 .^{\circ}$ de Bachillerato, es sensiblemente diferente- y Ciencias Aplicadas a la Actividad Profesional. En el caso de Cultura Científica de 4. ${ }^{\circ}$ de $\mathrm{ESO}$, los contenidos muestran un mayor paralelismo con la predecesora CMC de $1 .^{\circ}$ de Bachillerato (LOE), aunque, dado que se trata de una materia específica 
Figura 1. Metodología de análisis llevada a cabo en la investigación. Fuente: elaboración propia.

que comparte oferta con un amplio abanico de opciones, la probabilidad real de su impartición es baja.

\section{METODOLOGÍA}

En la presente investigación se ha analizado el currículo de las nuevas materias de la $\mathrm{LOE}(\mathrm{CMC})$ y la LOMCE (CC y AA) que resultan afines a la promoción de la competencia científica en Bachillerato. Tal y como recoge el esquema resumen de la figura 1, en primer lugar, se ha realizado un análisis cuantitativo, donde se explora la probabilidad de que un alumno tenga la posibilidad de cursar dichas materias. Y, en segundo lugar, un análisis cualitativo, en el que se han revisado y comparado los bloques de contenidos curriculares de las asignaturas mencionadas.

\section{AnÁlisis CUANTITATIVo}

Para calcular la probabilidad de que un alumno de Bachillerato tenga la opción de cursar alguna de las materias afines al desarrollo de la competencia científica, se han considerado tres aspectos de la arquitectura curricular: el carácter optativo u obligatorio de la materia, el número de materias que puede elegir cursar el alumno y la inclusión de estas (o no) en un itinerario concreto del Bachillerato.

En este sentido, es preciso recordar una de las modificaciones más significativas que introdujo la LOMCE en relación con el Bachillerato. Se trataba de la reestructuración de sus tipos de itinerarios especializados. Por un lado, si bien en el itinerario científico de la LOE (denominado "Ciencias y Tecnología») se ofertaban materias afines tanto al ámbito de las ciencias experimentales (Biología, Física, Química, Ciencias de la Tierra y Medioambientales...) como al de la tecnología (Dibujo Técnico, Electrotecnia, Tecnología Industrial...), el homólogo de la LOMCE se centra en las ciencias empíricas (BOE, 2014, artículos 27 y 28). La diferencia entre estos contextos es sutil, pero marca la exclusión de materias más próximas a la Formación Profesional, como pudiera ser la Electrotecnia, y al mismo tiempo reduce la multiplicidad en la oferta obligatoria real de optativas impartidas en los centros. 
Por otro lado, la LOMCE adopta una nueva configuración de las materias, que afecta no solo al Bachillerato, sino a todas las etapas educativas. Las materias se estructuran en tres grandes grupos: 1) las de carácter troncal, dentro de las cuales hay tanto materias obligatorias para todo el alumnado como optativas, propias del itinerario del Bachillerato; 2) las de configuración específica, que se dividen nuevamente en obligatorias y optativas, pudiendo elegir de estas últimas un mínimo de dos y un máximo de tres materias; y 3) las de libre configuración, dependientes de la administración educativa de cada Comunidad Autónoma, entre las cuales se incluyen también materias obligatorias y optativas. Tal y como actualmente queda reflejado, las materias de libre configuración pueden ser tanto materias de ampliación de contenidos de las asignaturas cursadas dentro de los bloques de troncales y de configuración específica como materias optativas no cursadas previamente, pero ofertadas dentro de este segundo grupo.

\section{AnÁlisis CUalitativo}

En este caso se han analizado los bloques de contenidos de CMC, CC y AA, recogidos en el Real Decreto 1467/2007 y el Real Decreto 1105/2014, documentos que establecen el currículo para la ESO y el Bachillerato de la LOE y la LOMCE, respectivamente. En este análisis se ha tratado de establecer una correlación entre los contenidos de las materias de la LOE y la LOMCE, con el fin de ver cuáles aparecen, se mantienen o desaparecen en la normativa vigente. En este sentido, es preciso matizar que la redacción de una nueva ley educativa generalmente no parte de tabula rasa sino que, en cierta medida, la ley o leyes anteriores sirven de sustrato para construir las siguientes. Por este motivo, no resulta ilógico plantear que la nueva materia de la LOMCE, CC, sea heredera - por lo menos parcialmente- de su predecesora inmediata-CMC (LOE)-.

Por otro lado, en el análisis del contexto curricular de la LOMCE destacan dos cuestiones: la organización de las materias curriculares y la inclusión de contenidos de tipo conceptual, actitudinal, procedimental y competencial. En el nuevo currículo cada materia incluye una descripción general de los bloques de contenidos desagregados en dos tipos de descriptores: los "Criterios de evaluación», más generales, y los «Estándares de aprendizaje evaluables», mucho más concretos. En su predecesora, sin embargo, los contenidos aparecían detallados en los bloques e incluían también sus correspondientes "Criterios de evaluación». Además, en la mayoría de las materias aparece la puesta en práctica de los conocimientos, mediante proyectos de investigación que necesariamente deben ser transversales.

\section{RESULTADOS Y DISCUSIÓN}

A continuación, se muestran los resultados de los análisis cuantitativo y cualitativo de las materias Ciencias para el Mundo Contemporáneo (CMC), Cultura Científica (CC) y Anatomía Aplicada (AA). 
Materias

obligatorias:

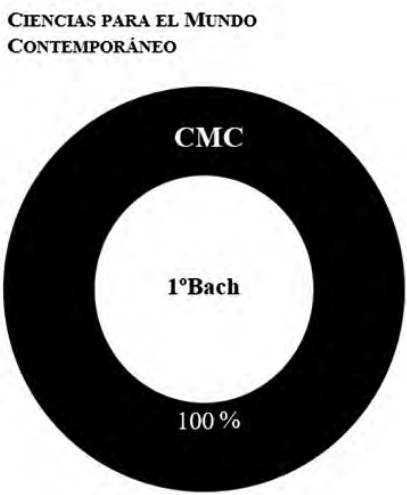

ESCENARIO:

Se oferta siempre: $100 \%$
Materias optativas de las especificas por itinerario:

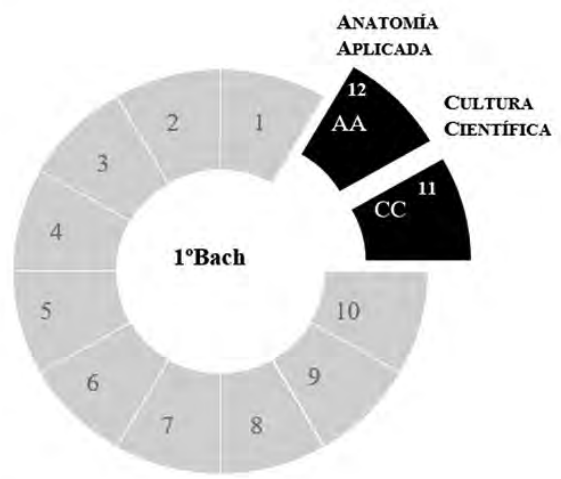

ESCENARIOS: Si se oferta..

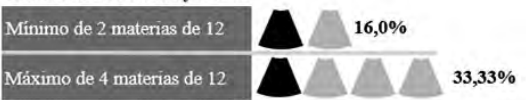

Figura 2. Reparto y probabilidad de impartición de materias optativas afines a la competencia científica en la LOE y la LOMCE. Fuente: elaboración propia.

\section{Análisis CUANTitativo}

En la figura 2 se recoge la probabilidad de impartición de las materias afines al desarrollo de la competencia científica en $10^{\circ}$ de Bachillerato en el contexto normativo de la LOE (CMC) y la LOMCE (CC y AA). Estas materias son comunes a todo el alumnado, con independencia del itinerario de Bachillerato que cursen (ver tabla 1, segundo apartado).

El primer aspecto destacable es el hecho de que CMC (LOE) era una materia obligatoria y, por lo tanto, la probabilidad de que fuera impartida era del $100 \%$. Sin embargo, con el cambio de marco jurídico (LOMCE) la probabilidad de impartición de CC y AA se ve reducida significativamente debido al carácter optativo de estas.

En segundo lugar, CC y AA comparten bloque con otras diez materias específicas, algunas de las cuales son muy habituales en los centros - como es el caso de Religión [católica]-, o tienen teórica continuidad en 2. ${ }^{\circ}$ de Bachillerato -como ocurre con Dibujo, Tecnología Industrial, Lenguaje Musical, o gozan en la actualidad de una gran demanda -como sucede con una segunda lengua extranjera (Francés o Alemán, habitualmente)-. El texto especifica que se tendrán que seleccionar entre 
un mínimo de dos materias y un máximo de tres, con lo que la probabilidad de que CC y AA se impartan puede variar entre el 16\% y el 33,33\% para cada una de ellas.

Sin embargo, considerando la alta probabilidad de impartición de materias tan sensibles como Religión [católica] o tan demandadas como los idiomas extranjeros, la posibilidad de que las asignaturas afines a la competencia científica analizadas se impartan definitivamente será significativamente menor. De hecho, el planteamiento más favorable en un escenario realista -teniendo presente tanto el perfil y especialidad del profesorado como la complejidad organizativa de la oferta docente en un centro de Educación Secundaria y Bachillerato- sería aquel en el que se incluyera una de las dos optativas dentro del amplio de abanico de propuestas. Por último, no se debe olvidar que, con independencia de si el centro oferta una materia o las dos, la probabilidad de que finalmente llegue o lleguen a cursarse depende, en última instancia, de la elección de asignaturas por parte del alumno, cuestión difícilmente medible o evaluable a priori, puesto que depende de otras cuestiones como las propias aspiraciones de este u otras tan volátiles como sus preferencias en relación con el docente que imparte la materia.

\section{AnÁlisis CUALITATIVo}

En relación con el análisis de los bloques de contenidos de CMC (LOE) y las nuevas materias de la LOMCE, en la tabla 2 se puede apreciar que casi la totalidad de cuestiones de CMC se repiten en la nueva materia de CC. De hecho, se aprecia un mismo orden tanto en la exposición como en la temática de estos bloques.

\begin{tabular}{|c|c|c|c|}
\hline Marco Legal & LOE (RD 1467/2007) & LOMCE (R & 1105/2014) \\
\hline Materias & $\begin{array}{l}\text { Ciencias para el Mundo } \\
\text { Contemporáneo (CMC) }\end{array}$ & $\begin{array}{l}\text { Cultura Científica } \\
\text { (CC) }\end{array}$ & $\begin{array}{l}\text { Anatomía Aplicada } \\
\text { (AA) }\end{array}$ \\
\hline $\begin{array}{l}\text { Análisis de con- } \\
\text { tenidos comunes } \\
\text { por bloques }\end{array}$ & $\begin{array}{l}\text { 1) La ciencia y sus procedi- } \\
\text { mientos } \\
\text { 2) Nuestro lugar en el universo } \\
\text { 3) Vivir más, vivir mejor } \\
\text { 4) Hacia una gestión sostenible } \\
\text { del planeta } \\
\text { 5) Nuevas necesidades, nuevos } \\
\text { materiales } \\
\text { 6) La aldea global. De la socie- } \\
\text { dad de la información a la } \\
\text { sociedad del conocimiento }\end{array}$ & $\begin{array}{l}\text { 1) Procedimientos de } \\
\text { trabajo (CMC 1,6) } \\
\text { 2) La Tierra y la vida } \\
\text { (CMC 2) } \\
\text { 3) Los avances en biome- } \\
\text { dicina (CMC 3) } \\
\text { 4) La revolución genética } \\
\text { (CMC 3) } \\
\text { 5) Nuevas tecnologías en } \\
\text { comunicación e infor- } \\
\text { mación (CMC 6) }\end{array}$ & $\begin{array}{l}\text { 1) Las características del } \\
\text { movimiento } \\
\text { 2) Organización básica del } \\
\text { cuerpo humano } \\
\text { 3) El sistema locomotor } \\
\text { 4) El sistema cardiopul- } \\
\text { monar } \\
\text { 5) El sistema de aporte y } \\
\text { utilización de energía } \\
\text { 6) Los sistemas de coordi- } \\
\text { nación y regulación } \\
\text { 7) Expresión y comunica- } \\
\text { ción corporal }\end{array}$ \\
\hline
\end{tabular}

Correspondencia entre los bloques de CMC y CC indicada entre paréntesis. Fuente: elaboración propia.

Al comparar los contenidos concretos recogidos en los bloques de CMC con los que previsiblemente se trabajarán en $\mathrm{CC}$-los cuales pueden deducirse a partir 
de los criterios de evaluación y los estándares de aprendizaje evaluables de la materia-, se aprecia que ambas materias comparten un gran número de temas de estudio. Sin embargo, como se puede o en la tabla 3, también hay diferencias entre ellas.

Un primer aspecto que llama la atención es que en CC se pierden algunos contenidos de naturaleza medioambiental que sí estaban presentes en CMC. Este es el caso del origen geológico de la Tierra, de la influencia del medio ambiente en la salud humana y de las consecuencias medioambientales del consumo de materiales (tabla 3, bloques 2, 3 y 5, respectivamente). Por tanto, en CC parecen afianzarse más las cuestiones biosanitarias (tabla 2, bloques 3 y 4), mientras que en CMC había un mayor equilibrio con los medioambientales (tabla 3 , bloques 4 y 5 ).

\begin{tabular}{|c|c|}
\hline Qué* DESAPARECE EN CC RESPECTO a CMC & QuÉ* APARECE EN CC RESPECTO A CMC \\
\hline $\begin{array}{l}\text { Bloque } 1 \text { CMC } \\
\text { Reconocimiento de las limitaciones y los errores de } \\
\text { la ciencia y la tecnología }\end{array}$ & $\begin{array}{l}\text { Bloque } 1 \text { CC } \\
\text { Estándares de aprendizaje evaluables: } \\
\text { 1.2) Busca información sobre ciencia y tecnología } \\
\text { tanto en soportes tradicionales como internet. } \\
\text { 3.1) Realiza comentarios analíticos de textos sobre } \\
\text { ciencia y tecnología y defiende en público sus } \\
\text { conclusiones. }\end{array}$ \\
\hline $\begin{array}{l}\text { Bloque } 2 \mathrm{CMC} \\
\text { El origen del universo } \\
\text { La formación de la Tierra }\end{array}$ & $\begin{array}{l}\text { Bloque } 2 \text { CC } \\
\text { Estándares de aprendizaje evaluables: } \\
\text { 7.1) Describe las últimas investigaciones científicas } \\
\text { sobre el origen y desarrollo de la vida en la Tierra }\end{array}$ \\
\hline $\begin{array}{l}\text { Bloque } 3 \text { CMC } \\
\text { La salud como resultado de los factores genéticos, } \\
\text { ambientales y personales. Estilos de vida saludables }\end{array}$ & $\begin{array}{l}\text { Bloque } 3 \text { CC } \\
\text { Estándares de aprendizaje evaluables: } \\
\text { 1) Conoce la evolución histórica de los métodos de } \\
\text { diagnóstico y el tratamiento de enfermedades. } \\
\text { 2.1) Distingue la medicina tradicional de la alterna- } \\
\text { tiva, valorando su fundamente científico y sus } \\
\text { riesgos } \\
\text { Bloque } 4 \text { CC } \\
\text { (nada) }\end{array}$ \\
\hline
\end{tabular}

Bloque 5 CMC

Necesidad, uso y problemática ambiental de los materiales (deforestación, residuos). Materiales

(No existe un bloque equivalente en CC)

naturales. Nuevos materiales y tecnologías

Bloque 6 CMC

Bloque 5 CC

(nada)

Avance tecnológico: beneficios y problemas (consumismo, dependencia, problemas de socialización, delitos informáticos...)

* La tabla no recoge literalmente los contenidos curriculares, sino un resumen de estos. Fuente: elaboración propia.

Una segunda cuestión reseñable es que en CC los contenidos sobre el desarrollo tecnológico y científico y sobre las nuevas tecnologías de la información y la comunicación adquieren gran protagonismo. Se busca que el alumno no solo sepa 


\section{LOE (2007)}

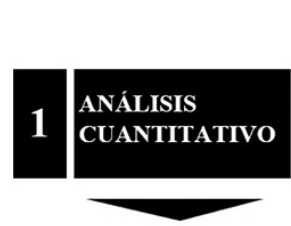

2

\section{ANÁLISIS \\ CUALITATIVO}

CIENCIAS PARA EL MUNDO

CONTEMPORÁNEO [CMC]

- Materia obligatoria para todos los itinerarios

- $100 \%$ impartición

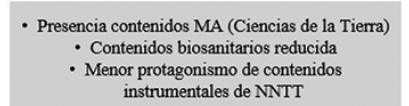

- Ausencia contenidos MA

Enriquecimiento de contenidos biosanitarios

- Mayor protagonismo de contenidos

instrumentales de NNTT

Figura 3. Análisis de resultados del estudio comparativo de las materias vinculadas a

la Competencia Científica LOE y la LOMCE (MA: Medioambientales;

NNTT: Nuevas Tecnologías). Fuente: elaboración propia.

qué es internet, sino que también conozca los riesgos y problemáticas asociados a su empleo (tabla 3, bloque 5 CC). En esta línea, también se pretende que el alumno sea capaz de valorar críticamente en base a una fundamentación científica cuestiones tan importantes como la efectividad y riesgos de las medicinas alternativas (tabla 3, bloque 3 CC). Se trata, en definitiva, de contenidos coherentes con las necesidades y demandas sociales y culturales actuales.

En lo que respecta a la nueva materia de AA, se trata de una materia con un marcado carácter biosanitario, pues está dedicada, de manera exclusiva, al estudio del funcionamiento del cuerpo humano. La asignatura busca la unión en la explicación de la motricidad, la anatomía, la fisiología, la biomecánica, así como las bases biológicas de las ciencias de la actividad física. En relación con los contenidos de la materia, los dos primeros bloques recogen las bases funcionales del movimiento. A estos siguen los elementos fundamentales de cada uno de los sistemas del organismo: sistema locomotor, cardiopulmonar, metabólico y de coordinación-regulación (bloques 3, 4, 5 y 6, respectivamente). Por último, en el bloque 7 se describen los aspectos de expresión y comunicación corporal, en los que se analiza el cuerpo como un elemento más del lenguaje.

La figura 3 recoge de forma esquemática la discusión resultante de los dos análisis comparativos efectuados en la presente investigación. En primer lugar, los vinculados a la tipología y estructura curricular en la que las distintas materias se integran y, en segundo lugar, los vinculados a los contenidos recogidos en cada una de las materias diseñadas dentro de cada uno de los marcos normativos.

\section{CONCLUSIONES}

La divulgación científica en la sociedad se ha convertido en una necesidad, tal y como lo atestiguan los especialistas y las administraciones en educación. El sistema educativo no puede ser indiferente a dicha demanda, puesto que es el medio 
en el que se construye el conocimiento de los futuros ciudadanos, a los cuales la ciencia prestará su servicio. Al mismo tiempo, la integración de contenidos de esta naturaleza dentro de los currículos supone la creación de un medio para establecer un foro de diálogo y reflexión, un canal adecuado para la alfabetización científica que reduzca el desigual acceso al conocimiento.

A lo largo de las últimas décadas la preocupación por la competencia científica ha aumentado, cuestión reflejada en la introducción creciente de contenidos de esta naturaleza en los currículos oficiales de las enseñanzas. La LOE en 2006 recogió con la asignatura generalista de Ciencias para el Mundo Contemporáneo (CMC) la necesidad impresa en el marco europeo. Dicha necesidad ha sido heredada en la LOMCE (2013) como parte de su preámbulo, pero con un desarrollo curricular y alcance muy diferente al anterior. La competencia científica se ha recogido en Bachillerato en dos nuevas asignaturas optativas: Cultura Científica (CC) y Anatomía Aplicada (AA).

A priori, podría pensarse que la competencia científica ha aumentado el abanico de actuación pasando de una asignatura en la LOE (CMC) a dos asignaturas en la LOMCE (CC y AA). Sin embargo, la aparición de dos nuevas materias no implica necesariamente una mayor oportunidad de desarrollo de la competencia científica. En este sentido es preciso tener en cuenta cuatro cuestiones:

1) El carácter optativo de las materias de la LOMCE. En contraposición a la obligatoriedad de CMC (LOE), en la LOMCE se reducen las posibilidades de que cualquier alumno (independientemente del itinerario que elija en Bachillerato) pueda cursar CC y AA. En este sentido, en el mejor de los casos, estas materias se ofertarán en los centros con una probabilidad máxima del 33,3\%.

2) La probabilidad real de que CC y AA se oferten en un mismo centro educativo. Las necesidades organizativas de los centros, del personal docente o de los propios agrupamientos condicionará la oferta de las materias optativas.

3) La probabilidad real de que el alumno elija esas materias. La elección de las materias optativas depende en última instancia de las decisiones individuales del alumnado.

4) El modo en que se potencie la competencia científica desde CC y AA. En último término, más allá de la oferta de materias específicas, el desarrollo de la competencia científica dependerá de las actividades que se empleen para trabajar los contenidos, de la metodología y las estrategias docentes utilizadas y de cómo se evalúe finalmente al alumnado.

En lo que respecta al análisis de contenidos de las materias descritas destacan dos cuestiones clave. Por un lado, el hecho de que la CC recogida en el actual marco legal se asienta y toma gran parte de los contenidos de su predecesora CMC, constituyendo una adaptación «actualizada» pero filtrada en lo que respecta al abanico de temas tratados. En segundo lugar, de forma más detallada, la revisión de contenidos de la materia de CC evidencia la desaparición de parte de los contenidos ambientales, mientras que la aportación de AA tiene un claro perfil biosanitario. En consecuencia, el saber científico se potencia en el Bachillerato de la LOMCE 
desde la perspectiva con mayor fraccionamiento y especialización. Se pierden contenidos medioambientales y se añaden contenidos biosanitarios.

La competencia científica resulta fundamental para sentar las bases conceptuales, pero, sobre todo, metodológicas indispensables no solo para el desarrollo del conocimiento experimental sino también para el desarrollo del espíritu crítico, fundamental para abordar de forma constructiva cuestiones complejas y asegurar el avance de la sociedad.

Al mismo tiempo, si bien existe un apoyo claro por parte de la comunidad científica, del profesorado y de las propias administraciones en incentivar el desarrollo de la educación competencial (dentro de la cual se enmarca la competencia científica), no deja de sorprender la cierta rigidez o incoherencia del diseño global de las etapas educativas del propio sistema, tanto en su versión previa, LOE, como en el actual. Como se ha expuesto, las Competencias -denominadas básicas primero y clave en la actualidad- se mantienen restringidas en las etapas obligatorias y no despliegan su continuidad de forma evidente en una etapa postobligatoria como es la del Bachillerato. Sin embargo, este periodo postobligatorio puede ser un contexto muy adecuado para aplicar un aprendizaje competencial debido al mayor nivel conceptual y la especialización de contenidos de la etapa. Además, es preciso recordar que el Bachillerato resulta fundamental para alcanzar los conocimientos y herramientas que darán sustento a los adquiridos posteriormente, tanto en la Formación Profesional como en la universitaria, donde nuevamente sí se retoma de forma clara y evidente la orientación educativa competencial.

Los cambios aquí recogidos son una muestra más del ir y venir de las propuestas y sistemas normativos educativos desarrollados a lo largo de la historia reciente de la educación en España. Muchos cambios periódicos que a menudo denotan una ausencia de acuerdo y cohesión, ambas cuestiones esenciales para una estabilidad y coherencia temporal y curricular. De hecho, parece que nuevamente nos encontramos en la antesala de una nueva normativa, ahora LOMLOE (Ley Orgánica de Modificación de la LOE). Solo el tiempo -y el consenso parlamentario- dirá qué ocurrirá finalmente con este proyecto. No se debe olvidar la complejidad metodológica que supone la comparación de los cambios curriculares, en ocasiones con contextos, clasificaciones y requerimientos muy diferentes. Así mismo, probablemente la principal dificultad radica en el escaso recorrido de gran parte de las leyes educativas, un breve margen temporal que impide un análisis fiable de las implicaciones que tiene sobre el alumnado, a medio y largo plazo, la inclusión de determinados contenidos en el currículo ordinario. Sin embargo, no cabe duda de que los cambios en las políticas educativas vinculadas a la promoción y divulgación de la competencia científica -con mayor o menor acierto-son fiel reflejo de la necesidad e interés de acercar la ciencia a la sociedad. 


\section{REFERENCIAS BIBLIOGRÁFICAS}

Acevedo, J. (1996). «Cambiando la práctica docente en la enseñanza de las ciencias a través de CTS». Borrador, 13, 26-30.

Acevedo, J. (1997). «Ciencia, Tecnología y Sociedad (CTS). Un enfoque innovador para la enseñanza de las ciencias». Revista de Educación de la Universidad de Granada, 10, 269-275.

Acevedo, J. (2004). «Reflexiones sobre las finalidades de la enseñanza de las ciencias: educación científica para la ciudadanía». Revista Eureka sobre Enseñanza y Divulgación de las Ciencias, 1(1), 3-16. Recuperado de http://venus.uca.es/eureka/revista/Volumen1/Numero_1_1/ Educa_cient_ciudadania.pdf.

Álvarez, F. y Fernández-Posse, G. (1996). Ciencia, tecnología, sociedad (Bachillerato LOGSE). Madrid: Laberinto.

Arroyo, J. (1999). «Material bibliográfico sobre ciencia, tecnología y sociedad. Bachillerato-LOGSE». Paideia: Revista de filosofía y didáctica filosófica, 20 (50), 521-532.

BOE (1990). Ley Orgánica 1/1990, de 3 de octubre, de Ordenación General del Sistema Educativo. Boletin Oficial del Estado n. ${ }^{\circ} 238$ del 4 de octubre de 1990. Recuperado de http://www. boe.es/boe/dias/1990/10/04/pdfs/A28927-28942.pdf.

$B O E$ (2006a). Ley Orgánica 2/2006, de 3 de mayo, de Educación. Boletín Oficial del Estado n. 106 del 4 de mayo de 2006. Recuperado de https://www.boe.es/boe/dias/2006/05/04/pdfs/ A17158-17207.pdf.

$B O E$ (2006b). Real Decreto 1513/2006, de 7 de diciembre, por el que se establecen las enseñanzas mínimas de la Educación primaria. Boletín Oficial del Estado n. ${ }^{\circ} 293$, del 8 de diciembre de 2006. Recuperado de http://www.boe.es/boe/dias/2006/12/08/pdfs/A43053-43102.pdf.

$B O E$ (2007a). Real Decreto 1631/2006, de 29 de diciembre, por el que se establecen las enseñanzas mínimas correspondientes a la Educación Secundaria Obligatoria. Boletín Oficial del Estado n. ${ }^{0} 5$, del 5 de enero de 2007. Recuperado de http://www.boe.es/boe/dias/2007/01/05/pdfs/ A00677-00773.pdf.

$B O E$ (2007b). Real Decreto 1467/2007, de 2 de noviembre, por el que se establece la estructura del bachillerato y se fijan sus enseñanzas mínimas. Boletín Oficial del Estado n. ${ }^{\circ} 266$, del 6 de noviembre de 2007. Recuperado de http://www.boe.es/boe/dias/2007/11/06/pdfs/A4538145477.pdf.

BOE (2015a). Real Decreto 1105/2014, de 26 de diciembre, por el que se establece el currículo básico de la Educación Secundaria Obligatoria y del Bachillerato. Boletín Oficial del Estado n. ${ }^{\circ} 3$ del 3 de enero de 2015. Recuperado de https:/www.boe.es/boe/dias/2015/01/03/pdfs/ BOE-A-2015-37.pdf.

$B O E$ (2015b). Orden ECD/65/2015, de 21 de enero, por la que se describen las relaciones entre las competencias, los contenidos y los criterios de evaluación de la educación primaria, la educación secundaria obligatoria y el bachillerato. Boletín Oficial del Estado n. ${ }^{\circ} 25$ del 29 de enero de 2015. Recuperado de https://www.boe.es/boe/dias/2015/01/29/pdfs/BOE-A-2015-738.pdf.

CEPE (2012). Aprender para el futuro. Competencias en educación para el Desarrollo Sostenible. Suiza: CEPE. Recuperado de https://www.unece.org/fileadmin/DAM/env/esd/ESD_Publications/Competences_Publication.pdf. 
CEPE (2013). Capacitar a los Educadores para un Futuro Sostenible. Génova, Italia: CEPE. Recuperado de http://www.unece.org/fileadmin/DAM/env/esd/ESD_Publications/Empowering_ Educators_for_a_Sustainable_Future_ENG.pdf.

Comisión Europea (2012). Informe Eurydice. El desarrollo de las competencias clave en el contexto escolar en Europa: desafíos y oportunidades para la politica en la materia. Luxemburgo: Oficina de Publicaciones de la Unión Europea. Recuperado de http://eacea.ec.europa.eu/education/eurydice/documents/thematic_reports/145ES.pdf.

De Pro, A. (2009). «El estudio de los materiales en los libros de texto de Ciencias para el mundo contemporáneo». Alambique: Didáctica de las ciencias experimentales, 59, 79-92.

DíAz, I. y García, M. (2011). «Más allá del paradigma de la alfabetización. La adquisición de Cultura Científica como reto educativo». Formación Universitaria, 4 (2), 3-14.

Fernández-GonzÁlez, M. (2008). «Ciencias para el mundo contemporáneo. Algunas reflexiones didácticas». Revista Eureka sobre Enseñanza y Divulgación de las Ciencias, 58(5), 185-199. Recuperado de http://revistas.uca.es/index.php/eureka/article/view/3755.

Fullan, M. (1994). «La gestión basada en el centro: un olvido fundamental». Revista de Educación, 304, 147-161. Recuperado de http://www.mecd.gob.es/dctm/revista-de-educacion/articulosre304/re3040600492.pdf?documentId=0901e72b81271021.

GiL, D. y Vilches, A. (2001). «Una alfabetización científica para el siglo xxi: obstáculos y propuestas de actuación». Investigación en la escuela, 43, 27-37. Recuperado de http://investigacionenlaescuela.es/articulos/43/R43_3.pdf.

Gómez, A. (2001). «El paisaje como tema transversal en el Diseño Curricular Base (DCB) de la Educación Obligatoria. La montańa como objeto de estudio». Biblio 3W. Revista Bibliográfica de Geografía y Ciencias Sociales, 267, en línea. Recuperado de http://www.ub.edu/ geocrit/b3w-267.htm.

IPPC (2015). Climate Change 2014. Synthesis Report. Contribution of Working Groups I, II and III to the Fifth Assessment Report of the Intergovernmental Panel on Climate Change. [Core Writing Team, R.K. Pachauri and L.A. Meyer (eds.)]. Suiza: IPPC. Recuperado de http://www.ipcc.ch/report/ar5/syr/.

López, J. (1998). «Ciencia, Tecnología y Sociedad: el estado de la cuestión en Europa y Estados Unidos». Revista Iberoamericana de Educación, 18, 41-68. Recuperado de http://rieoei.org/historico/oeivirt/rie18a02.htm.

Mas, V. (2006). «Las ciencias para la ciudadanía en Francia: un análisis de la propuesta francesa similar a la que se va a introducir en España». Alambique: Didáctica de las ciencias experimentales, 49, 30-41.

Martín, M., Nieda, J. y Pérez, A. (2008). «Las ciencias para el Mundo Contemporáneo, asignatura común del Bachillerato». Alambique: Didáctica de las ciencias experimentales, 56, 80-86.

Membiela, P. (2001). Enseñanza de las ciencias desde la perspectiva CTS. Madrid: Narcea ediciones.

Millar, R. y Hunt, A. (2006). «La ciencia divulgativa: una forma diferente de enseñar y aprender ciencia». Alambique: Didáctica de las ciencias experimentales, 49, 20-29.

OCDE (2011). PISA 2009. Informe Español. Madrid: Ministerio de Educación -Dirección General de Evaluación y Cooperación Territorial. Recuperado de http://www.mecd.gob.es/dctm/ ministerio/horizontales/prensa/notas/2010/20101207-pisa2009-informe-espanol.pdf?documentId=0901e72b806ea35a. 
PNUD (2016). Informe sobre Desarrollo Humano 2016; Desarrollo humano para todos. Nueva York: PNUD. Recuperado de http://www.hdr.undp.org/sites/default/files/hdr_2016_ report_spanish_web.pdf.

PNUMA (2012). GEO5: Perspectivas del Medio Ambiente Mundial. Medio Ambiente para el futuro que queremos. Recuperado de http://www.pnuma.org/geo/geo5/GEO\%205\%20ESPANOL\%202013\%20WEB.pdf.

Puig, J. y Casas, M. (2017). «El impacto ambiental: un despertar ético valioso para la educación». Teoría de La Educación, 29(1), 101-128. Recuperado de http://revistas.usal.es/-revistas_trabajo/index.php/1130-3743/article/view/teoredu291101128/17344.

Pedrinaci, E. (2006). «Ciencias para el mundo contemporáneo: ¿una materia para la participación ciudadana?» Alambique: Didáctica de las ciencias experimentales, 49, 9-19.

Pedrinaci, E. (2008). «¿Tiene sentido una materia como las Ciencias para el mundo contemporáneo?». Enseñanza de las ciencias de la tierra: Revista de la Asociación Española para la Enseñanza de las Ciencias de la Tierra, 16(1), pp. 9-16. Recuperado de http://www.raco.cat/index.php/ ECT/article/view/120981/166496\%20.

Pedrinaci, E. (2011). Las ciencias para el mundo contemporáneo en el Bachillerato. Física y Química. Complementos de formación disciplinar. Barcelona: Ministerio de Educación, Graó.

Pedrinaci, E. (2012). «El ejercicio de una ciudadanía responsable exige disponer de cierta competencia científica", en E. Pedrinaci (coord.), 11 ideas clave. El desarrollo de la competencia cientifica (15-37). Barcelona: Graó.

Redondo, M. y Gómez, E. (2006). «La cultura científica y las ciencias para el mundo contemporáneo». Anales de la Real Sociedad Española de Quimica, 3, 59-62.

Rocard, M., Csermely, P., Jorde, D., Lenzen, D., Walberg-Henriksson, J. y Hemmo, V. (2008). Enseñanza de las ciencias ahora: Una nueva pedagogía para el futuro de Europa. [Traducción del texto de la Comisión Europea: Science Education Now: A Renewed Pedagogy for the Future of Europe]. Alambique: Didáctica de las ciencias experimentales, 55, 104-117.

Solbes, J. y Vilches, A. (2002). «Visiones de los estudiantes de secundaria acerca de las interacciones Ciencia, Tecnología y Sociedad». Revista Electrónica de Enseñanza de las Ciencias, 1(2), 80-91. Recuperado de http://reec.uvigo.es/volumenes/volumen1/REEC_1_2_3.pdf.

TÁrraga, P. y De Pro, A. (2013). «Cuando hablamos del currículum de CMC, ¿̨todas las Comunidades Autónomas hablan de lo mismo?». Revista Eureka sobre Enseñanza y Divulgación de las Ciencias, 10(1), 11-29. Recuperado de http://revistas.uca.es/index.php/eureka/article/view/2792/2440.

Tiana, A. (2011). «Análisis de las competencias básicas como núcleo curricular en la educación obligatoria espańola». Bordón. Revista de Pedagogía, 63(1), 63-75. Recuperado de https://recyt. fecyt.es/index.php/BORDON/article/view/28905.

UNESCO (2006). Decenio de las Naciones Unidas de la Educación para el Desarrollo Sostenible (2005-2014). Plan de aplicación internacional. París: UNESCO. Recuperado de http:// unesdoc.unesco.org/images/0014/001486/148654so.pdf.

Vilches, A. (1994). «Las interacciones ciencia, técnica, sociedad. Selección bibliográfica temática». Enseñanza de las Ciencias, 12 (1), 112-120. Recuperado de https://www.uv.es/-vilches/documentos\%20enlazados/Biblio-com.pdf. 
